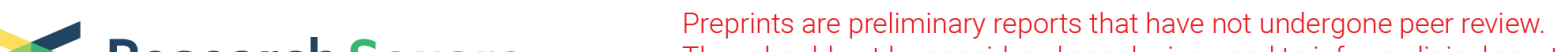 Research Square They should not be considered conclusive, used to inform clinical practice, or referenced by the media as validated information.
}

\section{MRI Radiomics to Differentiate Between Low Grade Glioma and Glioblastoma Peritumoral Region}

\section{Nauman Malik}

Sunnybrook Health Sciences Centre

\section{Benjamin Geraghty}

Sunnybrook Health Sciences Centre

\section{Archya Dasgupta}

Sunnybrook Health Sciences Centre

\section{Pejman Jabehdar Maralani}

Sunnybrook Health Sciences Centre

\section{Michael Sandhu}

Sunnybrook Health Sciences Centre Jay Detsky

Sunnybrook Health Sciences Centre

\section{Chia-Lin Tseng}

Sunnybrook Health Sciences Centre Hany Soliman

Sunnybrook Health Sciences Centre

Sten Myrehaug

Sunnybrook Health Sciences Centre

\section{Zain Husain}

Sunnybrook Health Sciences Centre

James Perry

Sunnybrook Health Sciences Centre

\section{Angus Lau}

Sunnybrook Health Sciences Centre

\section{Arjun Sahgal}

Sunnybrook Health Sciences Centre

Gregory Czarnota ( $\nabla$ gregoryczarnota.submissions@gmail.com )

Sunnybrook Health Sciences Centre https://orcid.org/0000-0002-0519-2182

\section{Research Article}


Keywords: Radiomics, Magnetic resonance imaging (MRI), Glioblastoma multiforme (GBM), Low grade glioma, Peritumoural region

Posted Date: August 3rd, 2021

DOl: https://doi.org/10.21203/rs.3.rs-758924/v1

License: (c) (1) This work is licensed under a Creative Commons Attribution 4.0 International License. Read Full License

Version of Record: A version of this preprint was published at Journal of Neuro-Oncology on October 25th, 2021. See the published version at https://doi.org/10.1007/s11060-021-03866-9. 


\section{Abstract \\ Background}

The peritumoral region (PTR) of glioblastoma (GBM) appears as a T2W-hyperintensity and is characterized by microscopic tumor and edema. Infiltrative low grade glioma (LGG) comprises tumor cells that seem similar to GBM PTR on MRI. The work here explored if a radiomics-based approach can distinguish between LGG and GBM PTR, which can have future implications on existing treatment paradigms.

\section{Methods}

Patients with GBM and LGG imaged using a 1.5 T MRI were included in the study. Image data from cases of GBM PTR, and LGG were manually segmented guided by T2W hyperintensity. A set of 91 first-order and texture features were determined from each of T1W-contrast, and T2W-FLAIR, diffusion-weighted imaging sequences. Applying filtration techniques, a total of 3822 features were obtained. Different feature reduction techniques were employed, and a subsequent model was constructed using four machine learning classifiers. Leave-one-out cross-validation was used to assess classifier performance.

\section{Results}

The analysis included 42 GBM and 36 LGG. The best performance was obtained using AdaBoost classifier using all the features with a sensitivity, specificity, accuracy, and area of curve (AUC) of 91\%, $86 \%, 89 \%$, and 0.96 , respectively. Amongst the feature selection techniques, the recursive feature elimination technique had the best results, with an AUC ranging from 0.87 to 0.92 . Evaluation with the Ftest resulted in the most consistent feature selection with $3 \mathrm{~T} 1 \mathrm{~W}$-contrast texture features chosen in over $90 \%$ of instances.

\section{Conclusions}

Quantitative analysis of conventional MRI sequences can effectively demarcate GBM PTR from LGG, which is otherwise indistinguishable on visual estimation.

\section{Introduction}

Radiomics is an emerging field in medicine and oncology involving quantitative feature analysis of highquality radiographic images $[1,2]$. Radiomics can provide information regarding tumor microenvironment and spatial, genomic, and proteomic data, which can be linked with prognosis, outcomes, and treatment response [3]. Magnetic resonance imaging (MRI) is considered the gold standard imaging modality for the management of central nervous system (CNS) malignancies, and it is particularly useful due to its 
high soft tissue contrast and the availability of different functional sequences, which can aid in tissue characterization [4]. Radiomic analysis has been widely undertaken in various CNS tumors, including gliomas, aiding in the differentiation between tumor histologies, grading, genetic profiling, and prognostication $[5,6]$.

Gliomas are a heterogeneous group of tumors arising from glial cells and named after their resemblance to glial cell lineages such as astrocytoma and oligodendrogliomas and classified into four grades that characterize biological behavior [7]. For the work here, we refer to grade 2 infiltrative gliomas as low-grade glioma (LGG), which typically are identified on MRI as T2-W hyperintense lesions. On MRI, distinct anatomical compartments can be identified in GBM, including tumor enhancement appearing as contrast-enhancing regions on T1-W sequences, with a central non-enhancing necrotic core and the adjacent peritumoural region (PTR) appearing as a T2-Weighted hyperintense area. Although the structural composition is quite distinct, the radiological appearance of LGG is similar to the PTR of GBM. In LGG, the areas of T2-W hyperintensity represent tumor, whereas GBM PTR is an admixture of microscopic infiltrative tumor and vasogenic edema $[8,9]$.

Our previous work demonstrated a radiomic-based approach from 270 features to categorize individual voxels in the PTR of GBM on a probabilistic scale to indicate infiltrative tumor or edema extrapolating feature characteristics from LGG and BM PTR, respectively [10]. The current work was undertaken to differentiate the entire region of GBM PTR from LGG, expanding the number of features (3822) using various filtration techniques. As opposed to 10 patients with GBM with post-operative MRI analyzed in the previous work, in this study, 42 patients with GBM were analyzed using preoperative index MRI. Also, in the present study, different feature selection techniques (all 270 features were used in the previous study for modeling) along with four machine learning classifiers (only support vector machine classifier in previous work) were investigated. The radiomics model can be explored in future studies to identify patches of microscopic disease within the PTR of GBM that can help reduce the clinical target volumes for radiation rather than treating the entire $\mathrm{T} 2 \mathrm{~W}$ signal changes.

\section{Methods}

\section{Patient Selection}

This retrospective study was approved by the institutional research ethics review board, and the requirement of obtaining consent was waived. Adult patients ( $\geq 18$ years of age) with IDH-wildtype GBM and LGG were identified from an institutional database from January 2014 to December 2018, with MRI data available from two identical scanners. For patients in the GBM cohort, preoperative MRI was used for analysis, usually undertaken within one week prior to the date of surgery. The LGG cohort included grade 2 astrocytoma and oligodendroglioma. Patients with high-risk or atypical features on histopathological evaluation or higher grade features on radiology, like intervening contrast-enhancing regions, significant T2W heterogeneity, were excluded. Post-operative scans were included in the study for the LGG cohort, but patients with prior radiotherapy or chemotherapy were excluded. During 
segmentation, the surgical cavity and tract were manually excluded. Tumors with segments smaller than $1 \mathrm{~cm}$ on at least one dimension were excluded from the study.

\section{MRI Protocol}

Imaging was acquired on a GE Signa HDxT 1.5T MRI scanner. using an 8-channel head coil (General Electric Medical Systems, Waukesha, WI, USA) was used. Three sequences were included in the current study: Gadolinium (Gd) (Gadovist $01 \mathrm{mmol} / \mathrm{kg}, 10 \mathrm{ml}$ maximum, bolus) enhanced 3D T1-weighted FSPGR (T1-CE); Gd-enhanced T2 fluid-attenuated inversion-recovery PROPELLOR (T2-FLAIR); and diffusion-weighted imaging-derived apparent diffusion coefficient maps (ADC). A single-shot echo-planar imaging sequence with 3 diffusion directions and a b-value of $1000 \mathrm{~s} / \mathrm{mm}^{2}$ was used to obtain diffusionweighted images, and ADC maps were reconstructed online using GE's Functool. The MRI acquisition parameters are shown in Supplementary Table 1.

\section{Image Preprocessing and Segmentation}

Figure 1 shows the schema used for the current study. The T2-FLAIR and ADC scans were first resampled to the corresponding T1-CE volume to match the field-of-view and resolution using the FMIRB Software Library (FSL) tool FLIRT. A pre-trained artificial neural network-based automated method was used for skull stripping using HD-BET. The extracted brain volumes from the T2-FLAIR scans were rigidly registered to the corresponding T1-CE volumes using FLIRT. For the ADC scans, the $b=0 \mathrm{~s} / \mathrm{mm}^{2}$ images were used for brain extraction and registration with the resulting transformations were applied to the ADC volumes. Data handling and scripting were performed in Matlab R2018b (The Mathworks, Inc., Natick, MA, USA). The T1-CE, T2-FLAIR, and ADC volumes were combined into single workspaces for each patient using the software platform ITK-SNAP (http://www.itksnap.org) for manual segmentation [11].

Segmentation was carried out manually initially by a radiation oncologist (AD), and all cases were individually reviewed by a neuroradiologist (PM) and a neuro-radiation oncologist (AS) in order to achieve final consensus. The segments in LGG included the tumor observed as T2-FLAIR hyperintensity, with the surgical cavity excluded for post-operative cases. In patients with GBM, the PTR was segmented to include the T2-FLAIR hyperintense region beyond the contrast-enhancing tumor core.

\section{Normalization \& Feature Extraction}

Prior to feature extraction, z-score intensity normalization was performed for all images by centering all pixel intensities within each brain to zero and then dividing by the standard deviation. The normalized images were then multiplied by 100 and shifted by 300 to ensure that the $\pm 3 \sigma$ majority of pixel intensities were non-negative. Fixed bin width (FBW) quantization was used to discretize pixel intensities within each segment $[12,13]$. The FBWs and corresponding bin counts (BC)s for the T1c images was $13\left(\mathrm{BC}_{\text {median }}=\right.$ $\left.52 ; \mathrm{BC}_{\text {range }}=16-87\right)$; for the T2f images was $20\left(\mathrm{BC}_{\text {median }}=27 ; \mathrm{BC}_{\text {range }}=16-43\right)$; and for the $\mathrm{ADC}$ images was $7\left(\mathrm{BC}_{\text {median }}=52 ; \mathrm{BC}_{\text {range }}=18-124\right)$. 
Feature extraction was performed using PyRadiomics software V2.2.0 The feature set included the following: 18 first-order statistical features; 22 gray level co-occurrence matrix (GLCM) features; 16 gray level size zone (GLSZM) features; 16 gray level run length matrix (GLRLM) features; 5 neighboring gray tone difference matrix (NGTDM) features; and 14 gray level dependence matrix (GLDM) features. Additional features were extracted by pre-processing images, either wavelet or Laplacian of Gaussian (LOG). LoG features were extracted with kernel sizes of 1, 2, 3, 4, and $5 \mathrm{~mm}$. All features were extracted from the segments in 3D. Pixel intensities outside the segments were set to zero prior to image filtration to reduce the effect of contamination. 91 features were derived from unfiltered images, 728 from wavelet filtered images and 455 from LoG filtered images, resulting in 1274 features per modality and a total of 3822 features. A detailed description of the features can be found on the Pyradiomics website (https://pyradiomics.readthedocs.io/en/latest/features.html).

\section{Feature Selection \& Classification}

All model building steps were performed in python using scikit-learn V0.22.2 [14]. Three feature selection approaches were utilized. Two were filter-based methods: ANOVA F-Test and minimum redundancy maximum relevance (mRMR). Recursive feature elimination (RFE), a wrapper-based approach, was also tested. A linear support vector machine (SVM) classifier (regularization parameter $C=1$ ) was used as the base learner, and at each iteration, the 5 least important features were eliminated from the total set until a pre-determined number of features was returned. For each feature selection method, sets of the top 4 features were included. This threshold was chosen based on the commonly employed rule-of-thumb that models should be trained on datasets that have at least 10 times as many training samples per class as the number of features to mitigate overfitting [15]. In order to demonstrate the impact of feature selection on classification performance, a fourth strategy used no feature reduction i.e. models were trained on all available features.

In order to prevent data leakage that can be present when the same samples used for feature selection are again used for model validation, a leave-one-patient-out (LOPO) cross-validation approach was utilized. This strategy precludes the identification of an "optimal" feature set, as different features can be selected as the training fold is permuted; however, it reduces the optimistic bias that can impact radiomics studies that make use of internal validation schemes. In lieu of identifying an "optimal" feature set, this approach can provide descriptive statistics of the selection frequency of different feature types and allow a degree of stability assessment with respect to feature selection. Features from the training data were scaled to zero mean and unit standard deviation at each LOPO iteration and the learned scaling parameters were applied to the features of the test patient.

Four machine learning classifiers were investigated in this study: support vector machine with a linear kernel (SVM); K-nearest neighbors (K-NN); linear discriminant analysis (LDA); and adaptive boosting using decision stumps as the base learner (AdaBoost). Hyperparameter selection was repeated at each LOPO cross-validation iteration through grid-search with nested 5-fold cross-validation to maximize balanced accuracy across the nested cross-validation procedure. For SVM, the range of the regularization 
parameter $\mathrm{C}$ was $10^{-4}$ to $10^{5}$ in multiples of 10 ; for $\mathrm{K}-\mathrm{NN}$, the range for the number of neighbors was from 1 to 11 in steps of 1 ; and for AdaBoost, the range for the number of trees was from 50 to 450 in steps of 100. All other tunable model hyperparameters were left as their default values assigned by scikit-learn (https://scikit-learn.org/0.22/). LOPO model performance was quantified by accuracy, sensitivity, specificity, and the area under the receiver operator characteristic curve (ROC AUC).

\section{Results}

A total of 74 patients were included in the analysis, 42 patients with GBM with preoperative scans with 42 tumors, and 32 patients with LGG without high-grade features on imaging with a total of 36 tumors. Patient characteristics are summarized in Table 1. The median patient age was 62 years for the GBM cohort (range 46-79) and 41 years for the LGG cohort (range 22-71), with the majority male patients (GBM 28/42, LGG 24/32). Twenty-five ( $n=25)(60 \%)$ of the GBM patients were $O(6)$-methylguanine-DNA methyltransferase (MGMT) methylated. In the LGG cohort, $25(69 \%)$ of patients had tumors with an astrocytic lineage, and 11 (31\%) had an oligodendrocytic lineage. In the LGG cohort, 27 (75\%) were IDH mutated by immunohistochemistry, and $10(28 \%)$ had $1 p / 19 q$ co-deletion detected.

\section{Classification}

A heat map of the performance metrics for each combination of feature selection method and classifier is presented in Figure 2, and the LOPO ROC curves are shown in Figure 3. Among the three feature selection methods tested, RFE resulted in the highest ROC AUC for all four classifiers $(0.868-0.916)$. The ANOVA F-test method resulted in the lowest accuracy scores for all classifiers (67.9-70.5). Since feature selection was performed independently at each LOPO iteration to prevent data leakage, the sets of selected features varied. With 4 selected features for each of the 74 LOPO iterations, the maximum number of unique features that could be selected was 296. Supplementary Figure 1 depicts a visual breakdown of the selected features by type across all LOPO iterations for each feature selection method. The majority of features selected were from T1-CE sequences (33-44\%), followed by ADC (33-39\%). The ANOVA F-test was the most stable method with respect to single patient permutation of the training set as only 9 unique features were selected, two of which were selected every time. In contrast, mRMR and RFE were less stable, with 62 and 46 unique features selected, respectively. Heat maps of the 5 most frequently selected features for each method along with their selection frequencies are shown in Figure 4. Differences were present between the GBM and LGG cases and are presented on a per case basis.

In the cases of model development without feature selection, i.e. all 3822 features included, the accuracy of the SVM classifier matched the highest score when feature selection was applied (83.3\%) while LDA (87.2\%) and AdaBoost (88.5\%) achieved their highest accuracies. Additionally, the highest ROC AUC for SVM, LDA, and AdaBoost was obtained when all features were used for training, with AUCs of 0.918, 0.915 , and 0.958 , respectively. In contrast, the K-NN methodology performed slightly worse in terms of both accuracy and ROC AUC as compared to the highest performance observed with feature selection. 


\section{Discussion}

Radiomics has the potential to integrate clinical information and outcomes with radiology using computer analytics and lead to the development of novel biomarkers in oncology. The PTR of GBM has been described as the area around contrast enhancement identified as hyperintense on T2 weighted and T2-FLAIR MRI sequences[16]. The complex microenvironment in the PTR region contains edema, vascular alterations, neoplastic cells, astrocytes, oligodendrocytes, inflammatory cells, microglia, stromal cells, pericytes [17], as well as GBM stem cells [18]. As approximately 90 percent of recurrences are witnessed in the PTR region [19], understanding tumor cell heterogeneity and microinvasion of cells in this region have the potential to impact treatment. Prasanna and colleagues [20] predicted survival outcomes using the top ten performing radiomic features in the PTR on routine MRI sequences. Despite the radiographic similarities on conventional MRI sequences, in contrast to GBM PTR, the architecture of LGGs is quite different. LGGs infiltrate surrounding brain tissue, but unlike GBM, they lack microvascular endothelial proliferation, necrosis and exhibit a lower Ki-67 proliferative index [21].

In this study, a radiomics approach using conventional MRI sequences distinguished GBM PTR from LGG with reasonable performance. Applying well-established classifiers with three feature selection algorithms to each of the sequences, T1-CE, T2-FLAIR, and ADC resulted in relatively high levels of discrimination. In terms of classification accuracy, performance ranged from $67.9 \%$ to $88.5 \%$ among the various models and feature selection approaches tested. Interestingly, the highest performing model was AdaBoost without using feature selection. The ROC AUC was also highest for SVM and LDA when all features were used for training as compared to models built using only 4 features. These models appear to handle high dimensional data as regularization and/or relative feature importance are implicit in their design. In contrast, the K-NN methodology performed worse when feature selection was not used prior to model training. K-NN strictly computes the distance of samples to its neighbors in the feature space and therefore assigns no relative importance to different features. When the number of features is large, the performance of local approaches such as K-NN is known to deteriorate ${ }^{34}$, which is encompassed in the phenomenon referred to as the "curse of dimensionality." A large number of features and the limited number of samples likely contributed to spurious associations between samples, which resulted in good specificity (86.1\%) but poor sensitivity $(69.0 \%)$. These results indicate that the risk overfitting attributable to feature dimension relative to sample size is both model and data-dependent and can be observed using robust cross-validation.

Distinguishing between LGG tumors and the PTR region of GBM has potential clinical applications. Previous work had demonstrated a radiomic model could demarcate brain metastasis peritumoural region (prototype for vasogenic edema) from LGG (prototype for infiltrative tumor) [10]. The model was extrapolated on the individual voxels within PTR of GBM to identify areas of microscopic tumor, which was shown to positively correlate with future areas of recurrence in a proportion of patients. In the present study, we had included a higher number of patients with GBM using preoperative MRI, a large number of features were generated, multiple feature selection methods, and four machine learning classifiers were used for model development. The current work can be expanded to independently identify 
microscopic disease in the GBM PTR using the LGG-like signature. Also, the radiomics model can be integrated with the previous work to improve the classification performances and increase the reliability of a combined model. Some studies have shown the potential improvement in clinical outcomes with surgical excision of the PTR region in GBM [22, 23], which is currently studied in a Canadian phase 2 randomized trial (NCT04737577). The present study will be particularly important in the preoperative identification of infiltrative microscopic disease since the images in the GBM cohort had preoperative MRI as opposed to post-operative imaging in the previous work. Similarly, identifying areas of infiltrative disease within the GBM PTR region can help in individualized radiation planning. Generally, radiation volumes include large margins empirically to include the entire $\mathrm{T} 2 \mathrm{~W}$ hyperintense areas without spatial information on the microscopic disease, which can lead to increased toxicity. In recent work, the use of small $5 \mathrm{~mm}$ margins beyond the gross tumor and cavity has shown survival outcomes similar to contemporary clinical trials [24]. The MRI-radiomics model will form an important basis in margin reduction in patients with GBM to be treated using an MRI-linear accelerator device (MR-LINAC) with GBM for a prospective phase 2 study of GBM (NCT04726397). Also, by identifying low grade disease within the PTR, this can allow differential dosing as low grade disease can be effectively treated with doses ranging from 45-54 Gy in 1.8-2.0 Gy per fraction, and the high grade components can be treated with 60 Gy in.1-8-2.0 Gy/day. This may reduce risks of radiation necrosis and help generating personalized clinical target volume delineation and dosing.

The work here has some limitations. This was a retrospective study with a limited sample size on one model of an MRI scanner. Future work can include the standardization of imaging features from different MRI scanners in order to validate the general applicability of such a model. Expansion of the study to include a larger number of patients, incorporating other MRI sequences, and undertaking external validation approaches will help establish a reliable and reproducible radiomics model in the future.

\section{Conclusion}

A radiomics model using routine MRI sequences can discriminate between low grade gliomas and the glioblastoma peritumoural region with acceptable performance, despite their visual resemblance. The best results were obtained using an AdaBoost classifier with an AUC of 0.96 to differentiate between the two groups.

\section{Declarations}

Presentation: The results of the study were presented at the $62^{\text {nd }}$ annual meeting of the American Society for Radiation Oncology (ASTRO) in October 2020.

Acknowledgments: We express our sincere gratitude to the patients and their caregivers involved in the study. We would like to thank the Terry Fox Foundation Program Project Grant from the Hecht Foundation for the funding support associated with the study. 
Funding: Terry Fox Foundation Program Project Grant from the Hecht Foundation (1083) awarded to Gregory J. Czarnota. The funding bodies had no influence on the study design, data collection, analysis, interpretation of data, or the manuscript's writing.

\section{Conflict of interest:}

Nauman Malik: None

Benjamin Geraghty: None

Archya Dasgupta: None

Pejman Maralani: None

Michael Sandhu: None

Jay Detsky: None

Chia-Lin Tseng: Travel accommodations/expenses \& honoraria for past educational seminars by Elekta, belongs to the Elekta MR-Linac Research Consortium, and advisor/consultant with Sanofi

Hany Soliman: None

Sten Myrehaug: Travel accommodations/expenses from Elekta AB. Research support from Novartis/AAA.

Zain Husain: Travel accommodations/expenses from Elekta.

James Perry: None

Angus Lau: None.

Arjun Sahgal: Advisor/consultant with AbbVie, Merck, Roche, Varian (Medical Advisory Group), Elekta (Gamma Knife Icon), BrainLAB, and VieCure (Medical Advisory Board)

Board Member: International Stereotactic Radiosurgery Society (ISRS)

Past educational seminars with Elekta AB, Accuray Inc., Varian (CNS Teaching Faculty), BrainLAB, Medtronic Kyphon

Research grant with Elekta $A B$

Travel accommodations/expenses by Elekta, Varian, BrainLAB

Elekta MR Linac Research Consortium, Elekta Spine, Oligometastases and Linac Based SRS Consortia

Gregory J. Czarnota: Funding received from the Terry Fox Foundation Program Project Grant. 
Availability of data and material: Data will be made available on request to the corresponding author following institutional ethics committee protocols.

Code availability: The radiomic feature extraction was performed using freely available Pyradiomics software (http://www.pyradiomics.io/pyradiomics.html). All standardization, model fitting, and assessment were performed using Scikit-Learn (https://scikit-learn.org/stable).

\section{Author contributions:}

Conceptualization: AD, BG, AS, GJC; Methodology: All authors; Formal Analysis and investigation: All authors; Writing-original draft preparation: NM, AD, BG, AS, GJC; Writing-review and editing: All authors; Project administration and supervision: AS, GJC; Funding acquisition: GJC

All the authors are in agreement and accountable for all the aspects of the work.

\section{References}

1. Lambin P, Rios-Velazquez E, Leijenaar R, et al (2012) Radiomics: Extracting more information from medical images using advanced feature analysis. Eur J Cancer 48:441-446. https://doi.org/10.1016/j.ejca.2011.11.036

2. Gillies RJ, Kinahan PE, Hricak H (2015) Radiomics: Images Are More than Pictures, They Are Data. Radiology 278:563-577. https://doi.org/10.1148/radiol.2015151169

3. Lambin P, Leijenaar RTH, Deist TM, et al (2017) Radiomics: the bridge between medical imaging and personalized medicine. Nat Rev Clin Oncol 14:749-762. https://doi.org/10.1038/nrclinonc.2017.141

4. Lundy P, Domino J, Ryken T, et al (2020) The role of imaging for the management of newly diagnosed glioblastoma in adults: a systematic review and evidence-based clinical practice guideline update. J Neurooncol 150:95-120. https://doi.org/10.1007/s11060-020-03597-3

5. Beig N, Bera K, Tiwari P (2020) Introduction to radiomics and radiogenomics in neuro-oncology: implications and challenges. Neurooncol Adv 2:iv3-iv14. https://doi.org/10.1093/noajnl/vdaa148

6. Lohmann P, Galldiks N, Kocher M, et al (2021) Radiomics in neuro-oncology: Basics, workflow, and applications. Methods 188:112-121. https://doi.org/10.1016/j.ymeth.2020.06.003

7. Louis DN, Perry A, Wesseling P, et al (2021) The 2021 WHO Classification of Tumors of the Central Nervous System: a summary. Neuro Oncol noab106. https://doi.org/10.1093/neuonc/noab106

8. Barajas RF, Phillips JJ, Parvataneni R, et al (2012) Regional variation in histopathologic features of tumor specimens from treatment-naive glioblastoma correlates with anatomic and physiologic MR Imaging. Neuro Oncol 14:942-954. https://doi.org/10.1093/neuonc/nos128

9. Eidel O, Burth S, Neumann J-O, et al (2017) Tumor Infiltration in Enhancing and Non-Enhancing Parts of Glioblastoma: A Correlation with Histopathology. PLoS One 12:e0169292. 
https://doi.org/10.1371/journal.pone.0169292

10. Dasgupta A, Geraghty B, Maralani PJ, et al (2021) Quantitative mapping of individual voxels in the peritumoral region of IDH-wildtype glioblastoma to distinguish between tumor infiltration and edema. J Neurooncol 153:251-261. https://doi.org/10.1007/s11060-021-03762-2

11. Yushkevich PA, Piven J, Hazlett HC, et al (2006) User-guided 3D active contour segmentation of anatomical structures: significantly improved efficiency and reliability. Neuroimage 31:1116-1128. https://doi.org/10.1016/j.neuroimage.2006.01.015

12. Duron L, Balvay D, Vande Perre S, et al (2019) Gray-level discretization impacts reproducible MRI radiomics texture features. PLoS One 14:e0213459. https://doi.org/10.1371/journal.pone.0213459

13. Carré A, Klausner G, Edjlali M, et al (2020) Standardization of brain MR images across machines and protocols: bridging the gap for MRI-based radiomics. Sci Rep 10:12340. https://doi.org/10.1038/s41598-020-69298-z

14. Pedregosa F, Varoquaux G, Gramfort A, et al Scikit-learn: Machine Learning in Python. MACHINE LEARNING IN PYTHON 6

15. Jain AK, Duin RPW, Mao J (2000) Statistical pattern recognition: a review. IEEE Transactions on Pattern Analysis and Machine Intelligence 22:4-37. https://doi.org/10.1109/34.824819

16. D’Alessio A, Proietti G, Sica G, Scicchitano BM (2019) Pathological and Molecular Features of Glioblastoma and Its Peritumoral Tissue. Cancers (Basel) 11:E469. https://doi.org/10.3390/cancers11040469

17. Sattiraju A, Mintz A (2019) Pericytes in Glioblastomas: Multifaceted Role Within Tumor Microenvironments and Potential for Therapeutic Interventions. Adv Exp Med Biol 1147:65-91. https://doi.org/10.1007/978-3-030-16908-4_2

18. Galli R, Binda E, Orfanelli U, et al (2004) Isolation and characterization of tumorigenic, stem-like neural precursors from human glioblastoma. Cancer Res 64:7011-7021. https://doi.org/10.1158/0008-5472.CAN-04-1364

19. Petrecca K, Guiot M-C, Panet-Raymond V, Souhami L (2013) Failure pattern following complete resection plus radiotherapy and temozolomide is at the resection margin in patients with glioblastoma. J Neurooncol 111:19-23. https://doi.org/10.1007/s11060-012-0983-4

20. Prasanna P, Patel J, Partovi S, et al (2017) Radiomic features from the peritumoral brain parenchyma on treatment-naïve multi-parametric MR imaging predict long versus short-term survival in glioblastoma multiforme: Preliminary findings. Eur Radiol 27:4188-4197. https://doi.org/10.1007/s00330-016-4637-3

21. Forst DA, Nahed BV, Loeffler JS, Batchelor TT (2014) Low-Grade Gliomas. Oncologist 19:403-413. https://doi.org/10.1634/theoncologist.2013-0345

22. Certo F, Altieri R, Maione M, et al (2020) FLAIRectomy in Supramarginal Resection of Glioblastoma Correlates With Clinical Outcome and Survival Analysis: A Prospective, Single Institution, Case Series. Oper Neurosurg (Hagerstown). https://doi.org/10.1093/ons/opaa293 
23. Jackson C, Choi J, Khalafallah AM, et al (2020) A systematic review and meta-analysis of supratotal versus gross total resection for glioblastoma. J Neurooncol 148:419-431.

https://doi.org/10.1007/s11060-020-03556-y

24. Azoulay M, Chang SD, Gibbs IC, et al (2020) A phase I/II trial of 5-fraction stereotactic radiosurgery with 5-mm margins with concurrent temozolomide in newly diagnosed glioblastoma: primary outcomes. Neuro Oncol 22:1182-1189. https://doi.org/10.1093/neuonc/noaa019

\section{Tables}

Table 1: Patient and disease charactersistics in the study 


\begin{tabular}{|ll|}
\hline Patient characteristics & \\
\hline Glioblastoma & 42 \\
\hline Number of patients & 42 \\
\hline Number of segments (lesions) & \\
\hline Age (years) & $62(46-79)$ \\
\hline Median (Range) & \\
\hline Gender & $28(67 \%)$ \\
\hline Male & $14(33 \%)$ \\
\hline Female & \\
\hline MGMT (GBM) & $25(60 \%)$ \\
\hline Methylated & $12(28 \%)$ \\
\hline Non-methylated & $5(12 \%)$ \\
\hline Unknown & \\
\hline Low Grade Glioma & $27(75 \%)$ \\
\hline Number of patients & $25(25 \%)$ \\
\hline Number of segments (lesions) & 36 \\
\hline Age (years) & $12(25 \%)$ \\
\hline Median (Range) & \\
\hline Gender & \\
\hline Male & \\
\hline Female & \\
\hline Histology (LGG) & \\
\hline Astrocytoma & \\
\hline Oligodendroglioma & \\
\hline IDH status (LGG) & \\
\hline Mutation & \\
\hline No mutation & \\
\hline Co-deleted & \\
\hline
\end{tabular}




$\begin{array}{ll}\text { No codeletion } & 21(58 \%) \\ \text { Unknown } & 5(14 \%)\end{array}$

\section{Figures}
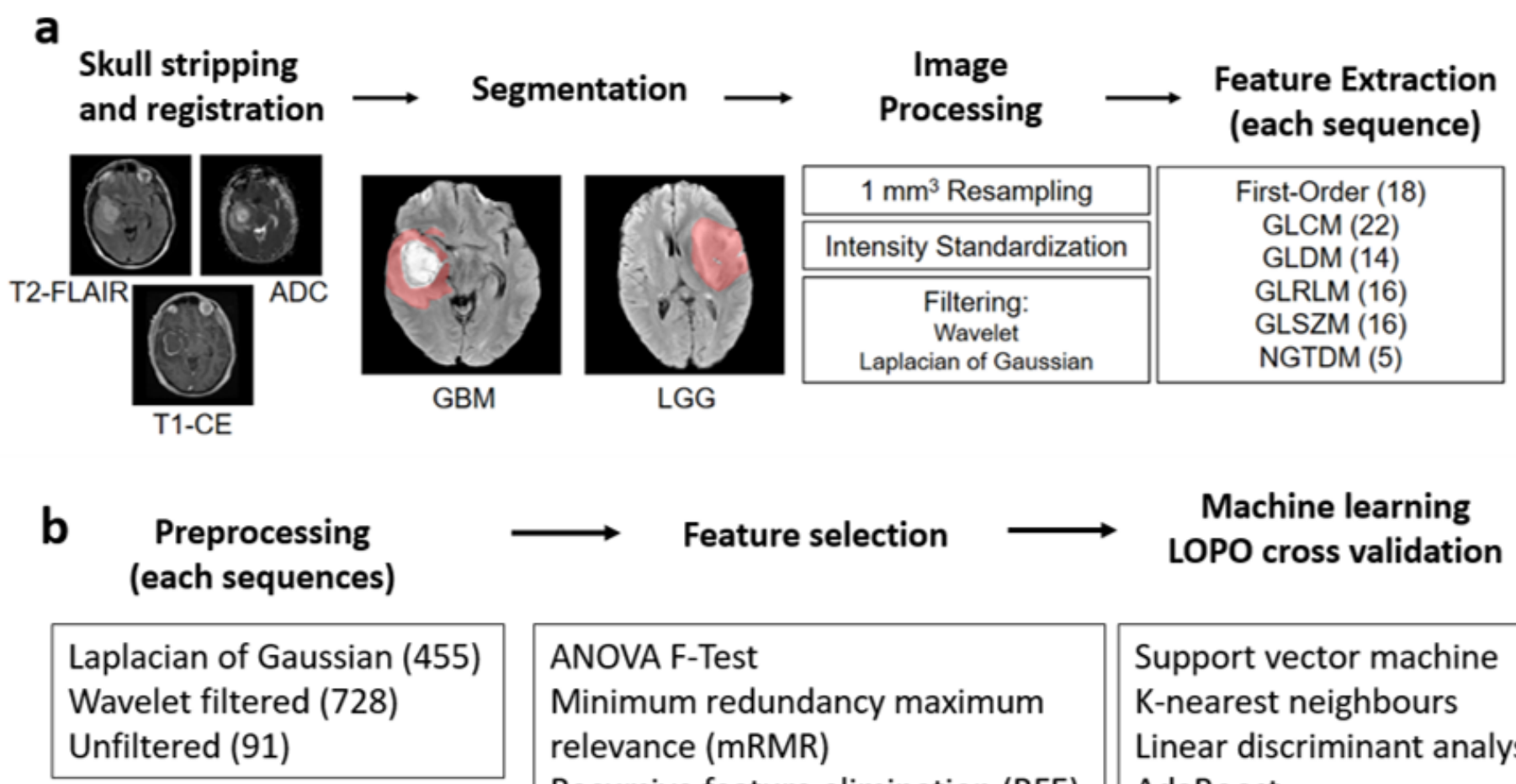

\section{ANOVA F-Test}

Minimum redundancy maximum

relevance (mRMR)

Recursive feature elimination (RFE)
Support vector machine

$\mathrm{K}$-nearest neighbours

Linear discriminant analysis

AdaBoost

Figure 1

Schematic of research methodology illustrating initial image registration, segmentation of the areas of interest in the two cohorts, glioblastoma peritumoral region (GBM PTR) and low-grade glioma (LGG), image processing, and feature extraction. 
Sensitivity

\begin{tabular}{c|c|c|c|c|}
\multicolumn{1}{c}{ F-Test } & \multicolumn{1}{c}{ mRMR } & \multicolumn{1}{c}{ RFE } & \multicolumn{1}{c}{ All } \\
\cline { 2 - 5 } SVM & 71.4 & 85.7 & 76.2 & 83.3 \\
\cline { 2 - 5 } k-NN & 69.0 & 81.0 & 83.3 & 69.0 \\
\cline { 2 - 5 } LDA & 76.2 & 83.3 & 81.0 & 85.7 \\
\cline { 2 - 5 } AdaBoost & 69.0 & 76.2 & 78.6 & 90.5 \\
\cline { 2 - 5 } & & &
\end{tabular}

Accuracy

\begin{tabular}{|c|c|c|c|c|}
\hline & F-Test & mRMR & RFE & All \\
\hline SVM & 70.5 & 83.3 & 80.8 & 83.3 \\
\hline k-NN & 67.9 & 78.2 & 82.1 & 76.9 \\
\hline LDA & 70.5 & 74.4 & 82.1 & 87.2 \\
\hline AdaBoost & 67.9 & 70.5 & 78.2 & 88.5 \\
\hline
\end{tabular}

Specificity

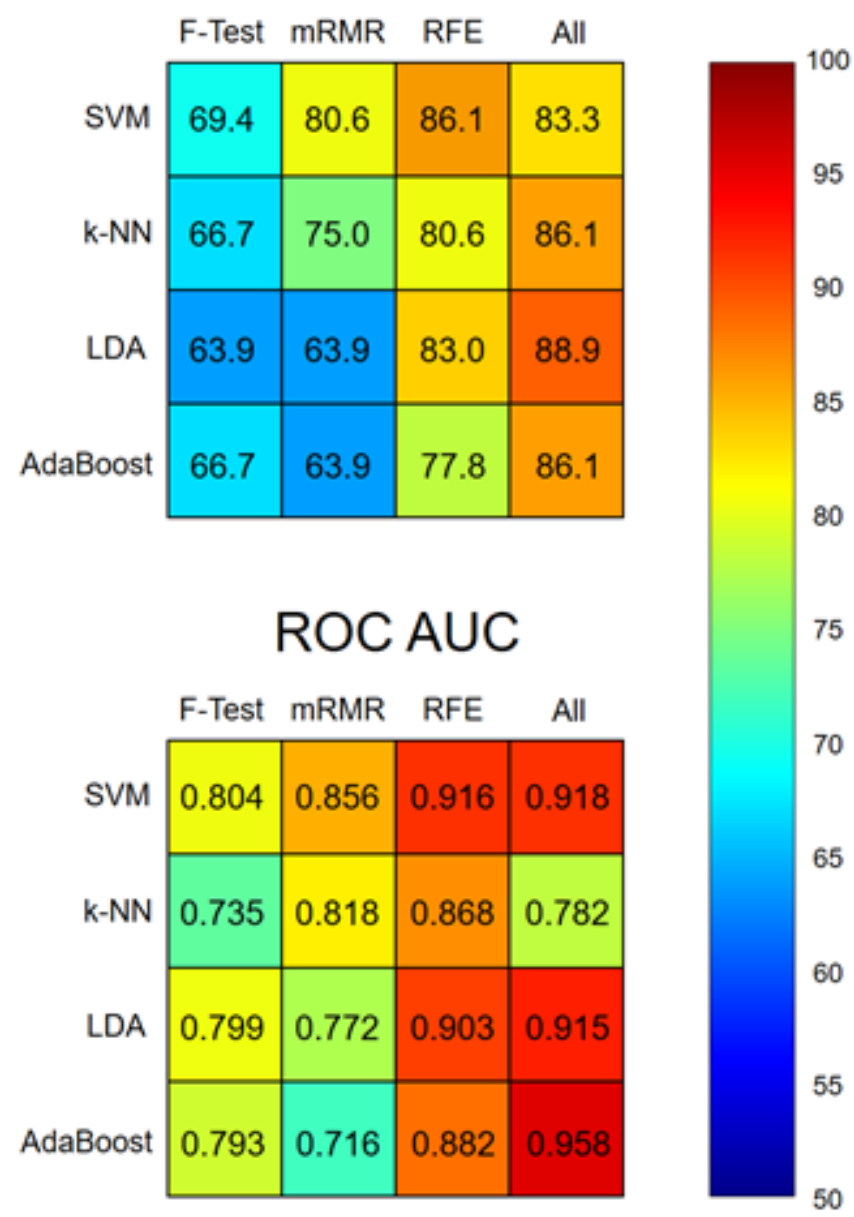

\section{Figure 2}

Accuracy, sensitivity, specificity, and receiver operating characteristic area under curve (ROC AUC) analyses with four classifiers, Support Vector Machine (SVM), K-Nearest Neighbors (K-NN), Linear Discriminant Analysis (LDA), and adaptive boosting (AdaBoost) for all extracted features. Three feature selection algorithms were applied: ANOVA F-test (F-Test), Minimum Redundancy Maximum Relevance (mRMR), and Recursive Feature Elimination (RFE). 

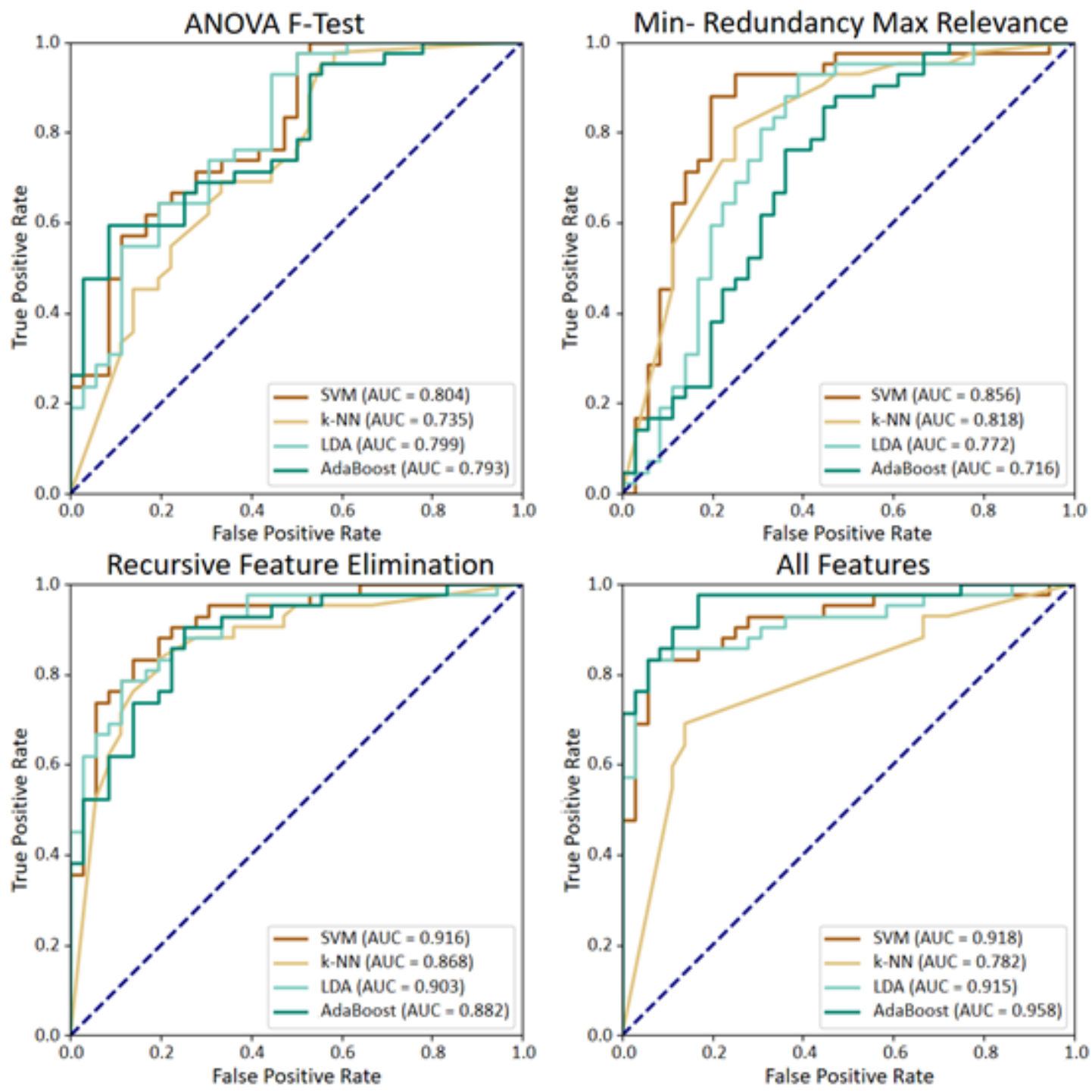

Figure 3

Receiver operating characteristic curves for each feature selection method using Support Vector Machine (SVM), k-Nearest Neighbors (K-NN), Linear Discriminant Analysis (LDA), and adaptive boosting (AdaBoost). 
a
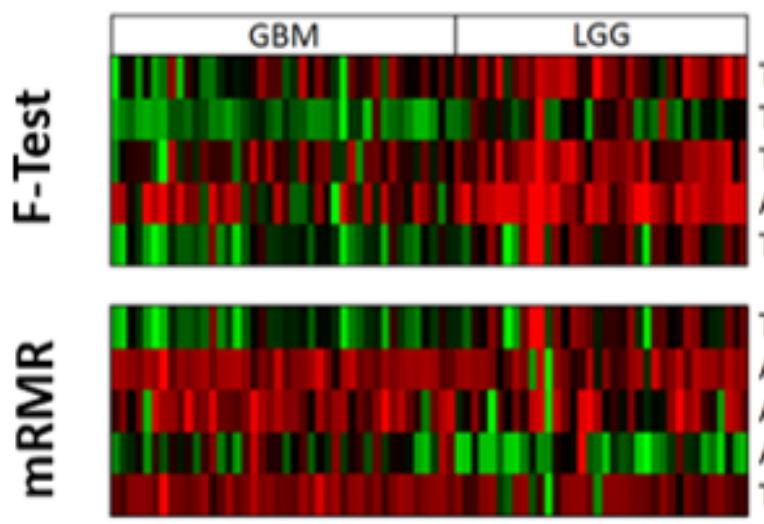

\section{AD}

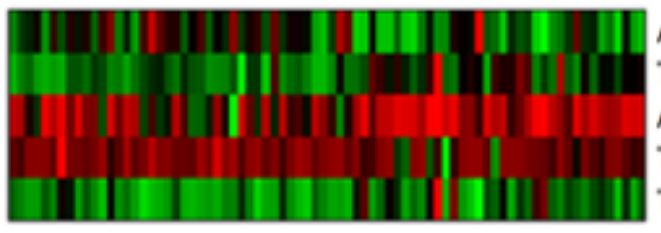

ADC_Unfiltered_GLCM_InverseVariance

T1-CE_Wavelet_HHL_GLSZM_ZoneEntropy ADC_Unfiltered_GLSZM_GrayLevelNonUniformity

T2-FLAIR_Wavelet_LLH_GLCM_ClusterShade

$-1$
T2-FLAIR_Unfiltered_GLSZM_ZoneEntropy

Selected Feature
T1-CE_Wavelet_LHL_NGTDM_Complexity
T1-CE_Wavelet_HHL_GLSZM_ZoneEntropy
T1-CE_Wavelet_HHL_NGTDM_Complexity
ADC_Unfiltered_GLSZM_GrayLevelNonUniformity
T1-CE_Wavelet_LHH_GLSZM_ZoneEntropy

1-CE_Wavelet_LHH_GLSZM_ZoneEntropy ADC_Wavelet_LLH_FirstOrder_Skewness

ADC_LoG_sigma_3_mm_GLCM_JointEnergy

ADC_Unfiltered_GLCM_InverseVariance

2-FLAIR_Wavelet_LLH_GLCM_ClusterShade

\section{Frequency \\ $100 \%$ \\ $100 \%$ \\ $93.2 \%$ \\ $68.9 \%$ \\ $25.7 \%$ \\ $98.6 \%$ \\ $51.4 \%$ \\ $37.8 \%$ \\ $21.6 \%$ \\ $20.3 \%$ \\ $55.4 \%$ \\ $37.8 \%$ \\ $36.5 \%$ \\ $24.3 \%$ \\ $23.0 \%$}

b

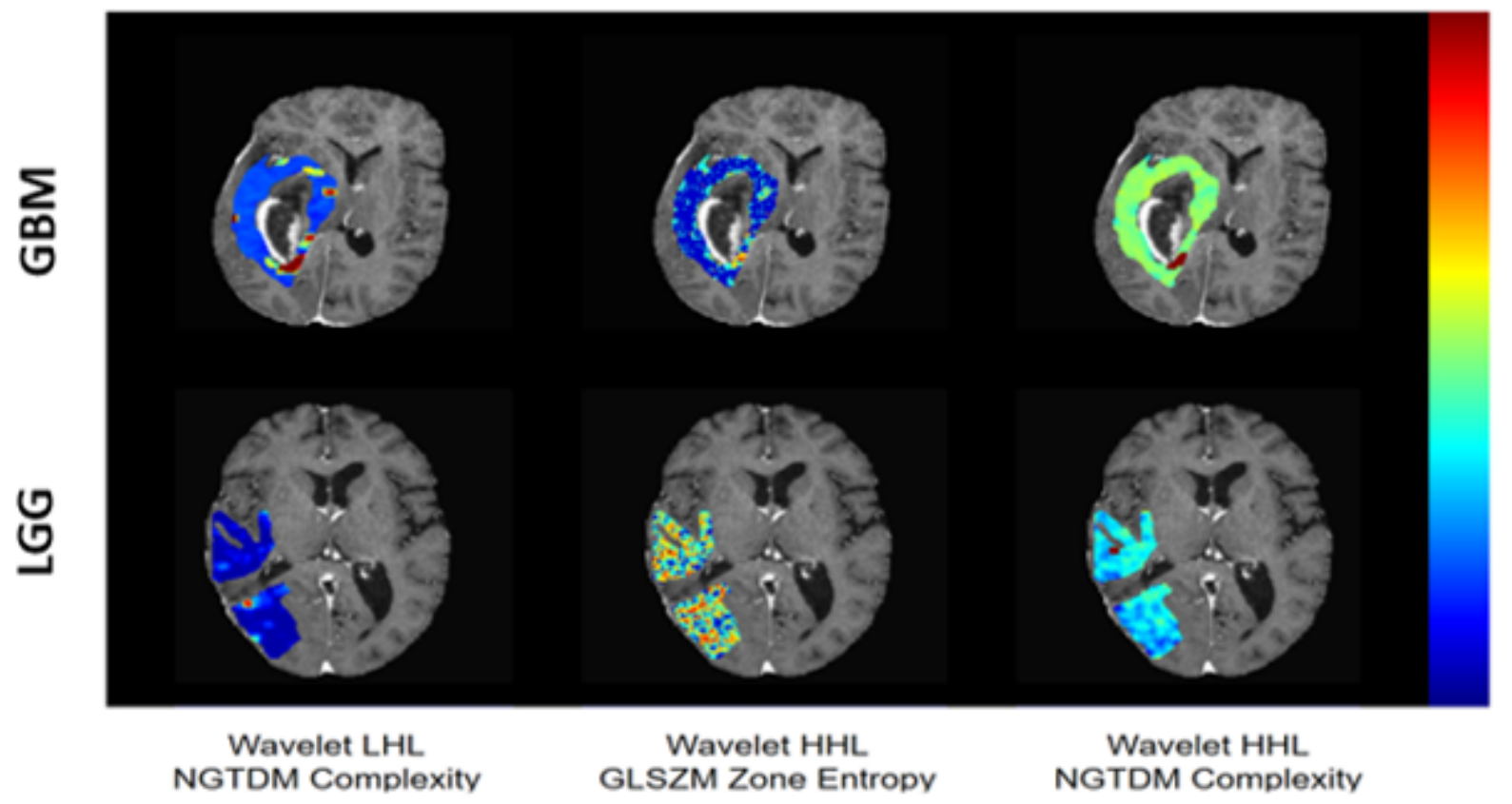

Figure 4

Figure 4a shows the heat map of the 5 most frequently selected features for each feature selection method across 74 iterations of leave-one-patient-out cross-validation. Feature values were scaled from -1 to 1 for visualization. Figure $4 \mathrm{~b}$ represents the parametric map with quantitative feature values in the regions of interest (GBM peritumoural region and low grade glioma) for the three most consistently selected features (all from T1W-contrast sequences) from the F-Test.

\section{Supplementary Files}


This is a list of supplementary files associated with this preprint. Click to download.

- SupplementaryFigure1.tif

- Supplementarytable1.docx 